\title{
AGLOMERADO AUTORREDUTOR DE RESIDUOS DE LD E ALTO FORNO: METALIZAÇÃO E EFEITO REGRIGERANTE *
}

\author{
Jerson Edwin Alvarado Quintanilla ${ }^{1}$ \\ Jose Carlos D'Abreu² \\ Edelink Efrain Tinoco Falero ${ }^{3}$ \\ Luiz Claudio Pinto Oliveira ${ }^{4}$
}

\section{Resumo}

$\mathrm{Na}$ indústria siderúrgica integrada, geram-se vários tipos de coprodutos, dentre estes escoria granulada de alto forno, os pós de aciaria extraídos nos sistemas de despoeiramento a úmido e a seco do conversor LD e o pó de coletor de alto forno. Em relação aos pós, são principalmente portadores de unidades de ferro e carbono, podendo conter também zinco, o que os tornam alvos de estudos e inovações que buscam recuperar ou reciclar estes elementos. No presente estudo foram concebidos briquetes autorredutores usando os pós finos-F e grossos - $\mathrm{G}$, oriundos de um sistema de despoeiramento a seco de aciaria LD, nas proporções percentuais, $\mathrm{F}-\mathrm{G}$, de 50-50 e 70-30, misturados com pó de coletor de alto forno. Utilizando como aglomerante o cimento ARI, foram avaliadas as resistências à compressão dos briquetes em diferentes tempos de cura e determinadas suas metalizações nas temperaturas de $1100^{\circ} \mathrm{C}$ e $1150^{\circ} \mathrm{C}$, em tempos que se estenderam até 45 minutos. Adicionalmente, as capacidades dos briquetes de alterarem a temperatura do aço liquido quando adicionados ao banho, foram avaliadas utilizando-se o balanço térmico do sistema briquete-aço liquido, bem como considerados os potenciais de oxidação do processo e o estabelecimento de um sequenciamento termodinâmico para as reações. Os resultados mostraram que os aglomerados autorredutores possuem alta capacidade refrigerante quando adicionados ao aço liquido, o que os enquadra como possíveis materiais alternativos à sucata e pelotas no controle da temperatura do aço nos convertedores LD. Além disso, a alta cinética de metalização do Fe mostrou ser também viável o uso dos briquetes autorredutores na recuperação do metal contido nos resíduos.

Palavras-chave: Resíduos; Briquetagem; Autorredução; Metalização.

\section{SELF-REDUCING AGGLOMERATES FROM LD AND BLAST FURNACE RESIDUES: METALLIZATION AND COOLING EFFECT}

\begin{abstract}
In the integrated steel industry several types of by-products are generated, such as, blast furnace slag, particulate residues from wet and dry LD dedusting systems and residues generated in the collector dust device of the blast furnace cleaning system. Containing in their compositions mainly iron and carbon units and in some cases also zinc, the iron and steelmaking dust and slurries have become target of various studies and technological initiatives, aiming to recover these elements. In this work the fine- $F$ and coarser- $G$ residues generated in a dry LD dedusting system, obeying two F-G proportion, 50-50 and 70-30, were mixed with the blast furnace dust and the ARI-cement and the final mixtures mechanically agglomerated to produce the selfreducing briquettes. The compression strength and the metallization degree at 1100 and $1150^{\circ} \mathrm{C}$ temperatures, were the main properties evaluated. Additionally, in order to know the briquettes cooling indexes when added in liquid steel, an energy balance for the briquette-steel bath system was calculated, taking into account a thermodynamic sequence for the reactions and the oxygen potentials of the process.
\end{abstract}


The self-reducing agglomerates showed to cause high cooling effect when added into a steel bath, being an alternative material to the scraps and iron ore pellets. Moreover, considering their high metallization degrees, the self-reducing briquettes also constitute an effective way to recover iron from residues.

Keywords: Residues; Briquetting; Self-reducing; Metallization

1 Eng. Metalúrgico, MSc, Doutorando, Departamento de Engenharia Química e de Materiais (DEQM), Pontifícia Universidade Católica do Rio de Janeiro (PUC-Rio), Rio de Janeiro, Brasil.

2 Eng. Metalúrgico, DSc, Professor Emerito, Departamento de Engenharia Química e de Materiais (DEQM), Pontifícia Universidade Católica do Rio de Janeiro (PUC-Rio), Rio de Janeiro, Brasil.

3 Eng. Metalúrgico, DSc, Pesquisador, Departamento de Engenharia Química e de Materiais (DEQM), Pontifícia Universidade Católica do Rio de Janeiro (PUC-Rio), Rio de Janeiro, Brasil

4 Eng. Metalúrgico, MSc, Engenheiro Senior, Companhia Siderúrgica do Pecém, Ceará, Brasil.. 


\section{INTRODUÇÃO}

A indústria siderúrgica é sabidamente uma atividade intensiva em utilização de energia, água e matérias-primas e caracterizada por elevadas gerações de gases, efluentes, resíduos e coprodutos, a cujos problemas ambientais decorrentes se contrapõem grandes esforços buscando minimizá-los e incrementar seus aproveitamentos e reciclagens, mirando essencialmente o desenvolvimento de tecnologias especificas que aumentem a geração de coprodutos. As Siderúrgicas integradas geram em média cerca de $600 \mathrm{~kg}$ de resíduos e coprodutos de processo para cada tonelada de aço, dos quais aproximadamente $70 \%$ são escórias [1]. $\mathrm{Na}$ etapa de despoeiramento primário das aciarias LD se destacam os sistemas "úmido" e "sêco", os quais geram dois resíduos característicos: os grossos (G), ricos em Fe metálico, representando cerca de $30 \%$, e os restantes $70 \%$, mais finos $(F)$, que concentram majoritariamente as fases oxidicas. $O$ índice médio de geração total desses particulados é de até $24 \mathrm{~kg} / \mathrm{t}$ aço [2]. Os aproveitamentos desses resíduos se fazem principalmente via as sinterizações e, em particular os gerados nos sistemas a úmido, via briquetes para a reciclagem do Fe contido [3,4] em processos tradicionais e alguns emergentes, além de, nas próprias aciarias, como material refrigerante alternativo adicionado para o controle da temperatura do aço liquido [5]. Em relação à esta última finalidade, alguns estudos experimentais já mostraram um efeito refrigerante relativamente vantajoso dos aglomerados autorredutores [6,7].

Em razão da natureza distinta dos particulados gerados pelos sistemas de despoeiramento a seco e a úmido do LD, foi estabelecido um programa cooperativo para estudar e definir alternativas para a reciclagem dos materiais $\mathrm{G}$ e $\mathrm{F}$ gerados pela rota seca. Como parte deste programa, o presente trabalho aborda a fabricação de aglomerados autorredutores constituídos pelos materiais $G, F$, nas proporções 50-50 e 30-70, respectivamente, e o resíduo carbonoso proveniente do coletor de pó do alto forno, com $10 \%$ de excesso. Após o período de cura, os briquetes foram testados à compressão e em seguida metalizados nas temperaturas de 1100 e 1150 ${ }^{\circ} \mathrm{C}$. Finalmente, para a determinação do poder refrigerante dos briquetes uma rota para as reações foi proposta em bases termodinâmicas e adotada como pre requisito para o desenvolvimento do balanço de energia do sistema briquete-aço liquido.

O presente trabalho apresenta os resultados obtidos no ensaio de resistência a compressão dos briquetes fabricados a partir do pós de aciaria ( $F-G)$ nas proporções percentuais de 50-50 e 70-30, com o pó de coletor de alto forno (10 \% em excesso), empregando cimento ARI como aglomerante, visando suas resistências em diferentes tempos de cura [8,9]. Também foram estudadas, as metalizações dos briquetes autorredutores, pó de coletor no estequiométrico e $10 \%$ excesso, em ensaios de redução nas temperaturas de $1100^{\circ} \mathrm{C}$ e $1150^{\circ} \mathrm{C}$. E finalmente o cálculo teórico da viabilidade de uso destes briquetes autorredutores como meios refrigerantes para o controle da temperatura do aço líquido nos convertedores LD, enquadrando-os, desta forma, como materiais reais e potencialmente alternativos à sucata e as pelotas e como carga ferrosa nos processos de redução visando à recuperação do elemento ferro dos resíduos. 


\section{MATERIAIS E MÉTODOS}

Os seguintes materiais foram utilizados para a fabricação dos aglomerados autorredutores, cujas composições e granulometria estão indicadas nas tabelas 1, 2, 3 e 4:

Tabela 1 Análise química do pó de aciaria LD

\begin{tabular}{cccccccccccc}
\hline Pó de aciaría & $\mathbf{F e}_{\text {total }}$ & $\mathbf{F e}_{\text {met }}$ & $\mathrm{SiO}_{2}$ & $\mathbf{C a O}$ & $\mathbf{M g O}$ & $\mathbf{N a}_{2} \mathbf{O}$ & $\mathbf{K}_{2} \mathbf{O}$ & $\mathbf{Z n}$ & $\mathbf{A L}_{2} \mathbf{O}_{3}$ & $\mathbf{C}$ & $\mathbf{S}$ \\
\hline Fino (\%) & 58,45 & 17,55 & 1,91 & 11,31 & 1,91 & 0,09 & 0,40 & 0,07 & 0,25 & 0,60 & 0,07 \\
\hline Grosso (\%) & 58,13 & 42,55 & 3,12 & 18,34 & 3,16 & 0,03 & 0,18 & 0,06 & 0,30 & 0,64 & 0,05 \\
\hline
\end{tabular}

Tabela 2 Análise granulométrica do pó de aciaria LD

\begin{tabular}{c|cccccc}
\hline \multirow{2}{*}{ Pó de aciaria } & \multicolumn{7}{|c}{ Retida na malha } \\
\cline { 2 - 7 } & $\mathbf{2} \mathbf{~ m m}$ & $\mathbf{1 ~} \mathbf{~ m m}$ & $\mathbf{0 , 3 5 5} \mathbf{~ m m}$ & $\mathbf{0 , 1 2 5} \mathbf{~ m m}$ & $\mathbf{0 , 0 4 5} \mathbf{~ m m}$ & Fundo \\
\hline Fino & 8,62 & 3,99 & 8,27 & 17,1 & 21,75 & 40,28 \\
\hline Grosso & 2,65 & 4,92 & 19,63 & 36,25 & 20,51 & 16,04 \\
\hline
\end{tabular}

Tabela 3 Análise química do pó de coletor de Alto Forno

\begin{tabular}{cccccccccccc}
\hline $\begin{array}{c}\text { Pó de Alto } \\
\text { Forno }\end{array}$ & $\mathrm{Fe}_{\text {total }}$ & $\mathrm{Fe}_{\text {met }}$ & $\mathrm{SiO}_{2}$ & $\mathbf{C a O}$ & $\mathbf{M g O}$ & $\mathrm{Na}_{2} \mathrm{O}$ & $\mathrm{K}_{2} \mathrm{O}$ & $\mathbf{Z n}$ & $\mathrm{AL}_{2} \mathrm{O}_{3}$ & $\mathbf{C}$ & $\mathbf{S}$ \\
\hline Coletor (\%) & 41,49 & - & 9,07 & 2,90 & 0,50 & 0,18 & 0,26 & 0,21 & 2,64 & 32,84 & 0,29 \\
\hline
\end{tabular}

Tabela 4 Análise granulométrica do pó de coletor de Alto Forno

\begin{tabular}{ccccccc}
\hline \multirow{2}{*}{ Pó de Alto Forno } & \multicolumn{6}{c}{ Retida na malha } \\
\cline { 2 - 7 } Coletor & $\mathbf{2} \mathbf{~ m m}$ & $\mathbf{1} \mathbf{~ m m}$ & $\mathbf{0 , 3 5 5} \mathbf{~ m m}$ & $\mathbf{0 , 1 2 5} \mathbf{~ m m}$ & $\mathbf{0 , 0 4 5} \mathbf{~ m m}$ & Fundo \\
\hline
\end{tabular}

Como os pós do despoeiramento primários são gerados a seco, foi necessária sua prévia estabilização, a fim de serem evitadas trincas e rupturas precoces nos briquetes. Considerando a importância da reatividade da fase carbonosa do briquete para o comportamento cinético das reações de redução e metalização [10], a medida desta propriedade empregou as condições da norma ASTM (D-5341) [11] e o ensaio TGA de perda de massa, nas condições de $1100^{\circ} \mathrm{C}$ e fluxo de $\mathrm{CO}_{2}$, durante 2h.

Após a passivação, passou-se à operação de mistura dos materiais $F$ e $G$, nas proporções 50-50 e 70-30, respectivamente, com uma quantidade de pó de coletor do AF expressando o valor estequiométrico e com $10 \%$ de excesso, para os ensaios de compressão e de metalização. Como aglomerante foi utilizado o cimento ARI, na proporção de 6\%, obedecendo à relação água/cimento de 0,5. As misturas, previamente umidificadas, eram briquetadas em prensa hidráulica, e os briquetes cilíndricos, com cerca de $8 \mathrm{~g}$, curados por até 28 dias. Amostras foram retiradas após 3, 8, 18 e 28 dias de cura e testadas à compressão. Para a metalização, realizada numa linha de gases com temperatura, pressão e atmosfera controladas, utilizou-se as amostras curadas até 28 dias. As figuras 1 e 2 apresentam a prensa e a linha de gases utilizados nas experiências. 


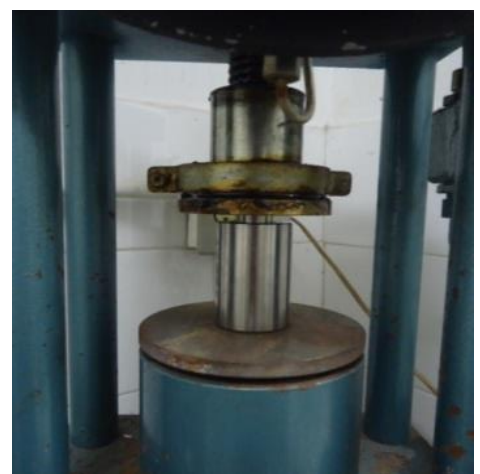

Figura 1 Prensa hidráulica

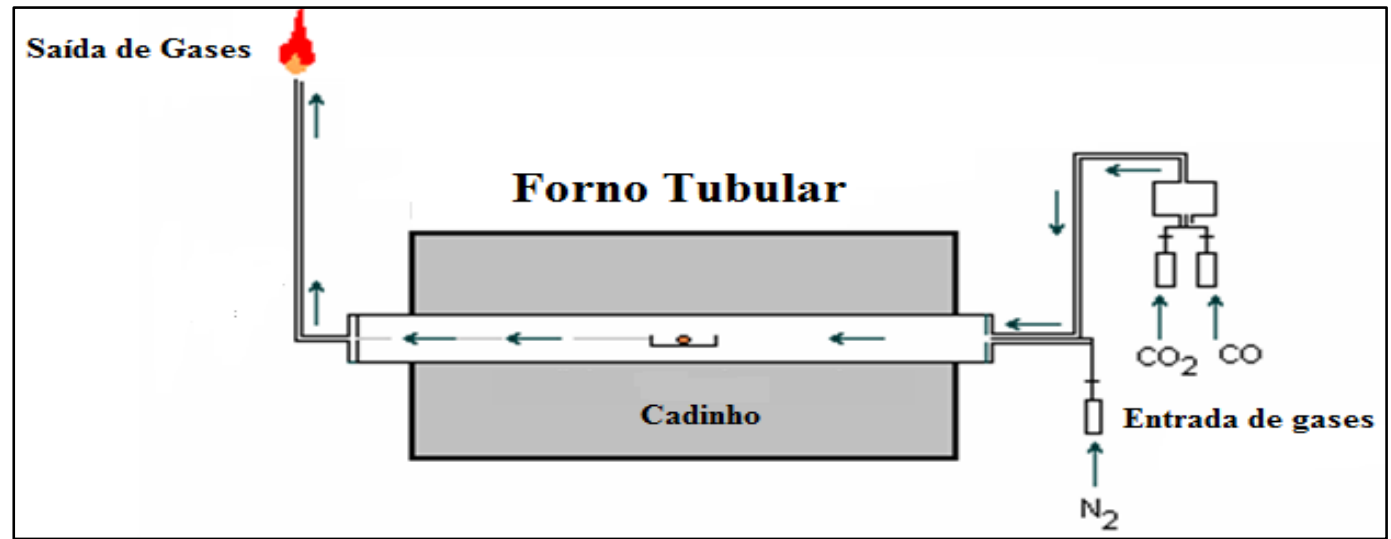

Figura 2 Linha de gases

Os ensaios de metalização foram realizados sob fluxos contínuos de $\mathrm{N}_{2}$, nas temperaturas de $1100^{\circ} \mathrm{C}$ e $1150^{\circ} \mathrm{C}$, procedendo-se a amostragens nos tempos de 5 , $10,20,30$ e 45 minutos. Os briquetes selecionados eram encaminhados para a determinação dos seus índices de metalização e, posteriormente, realizadas as análises cinéticas correspondentes, visando a definição do mecanismo reacional de melhor ajuste e em condições de aplicação no modelamento matemático.

\section{RESULTADOS E DISCUSSÃO}

\subsection{Hidratação dos pós da aciaria LD}

A Figura 3 ilustra os ganhos de massa dos pós de aciaria fino e grosso ao longo de 10 dias de hidratação, mostrando uma estabilização do processo, a partir do terceiro dia para o pó grosso e, no caso do pó fino, a partir do quarto dia. 


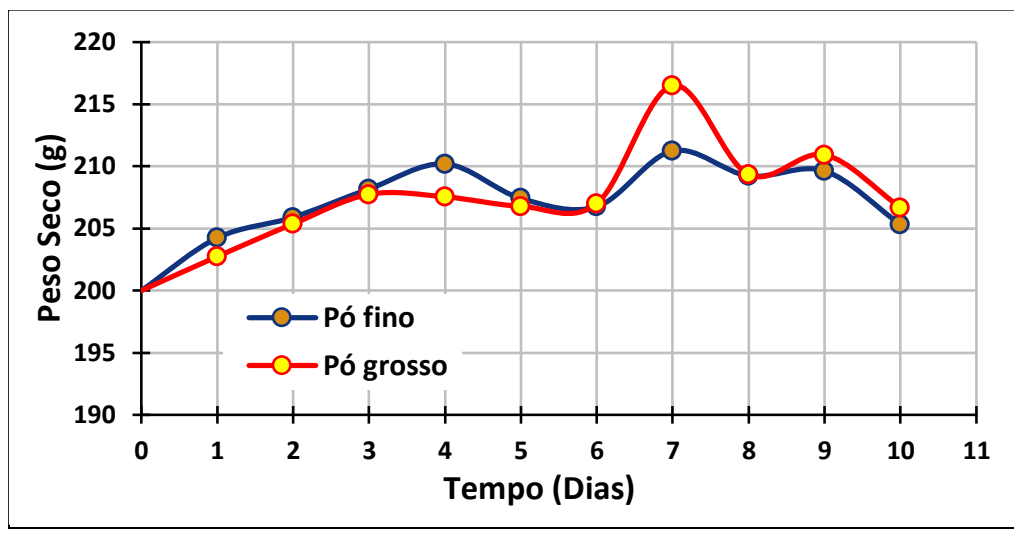

Figura 3 Passivação dos pós de aciaria: frações fina e grossa

\subsection{TGA dos pós de aciaria, após passivação.}

Na Figura 4(a), observa-se que o pó de aciaria, fração grossa, apresentou uma perda de massa equivalente a $4,02 \%$, na faixa de temperatura $21-882^{\circ} \mathrm{C}$, e o pó de aciaria fração fina, Figura 4(b), uma perda de massa equivalente a $6,34 \%$ na faixa de temperatura de 21 até $861^{\circ}$. Tais perdas foram compatíveis com os ganhos alcançados pelo processo de passivação.
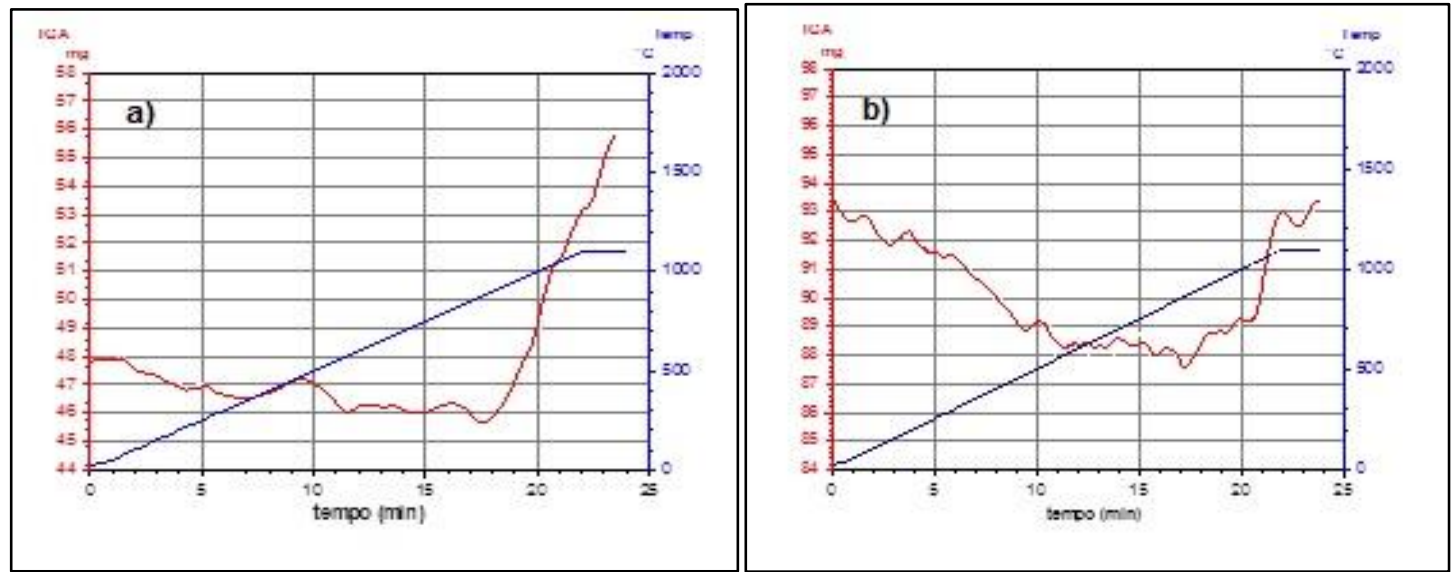

Figura 4 Termograma do pó de aciaria: a) fração Grossa; b) fração fina.

\subsection{Reatividade (ASTM e TGA)}

O teste de reatividade segundo a norma ASTM (D-5341) mostrou um valor médio alto, de $77,1 \%$, tendo em vista sua granulação fina. Utilizando o TGA (Figura 5) a reatividade média alcançou 53\%, sendo este o valor adotado para esta propriedade. 


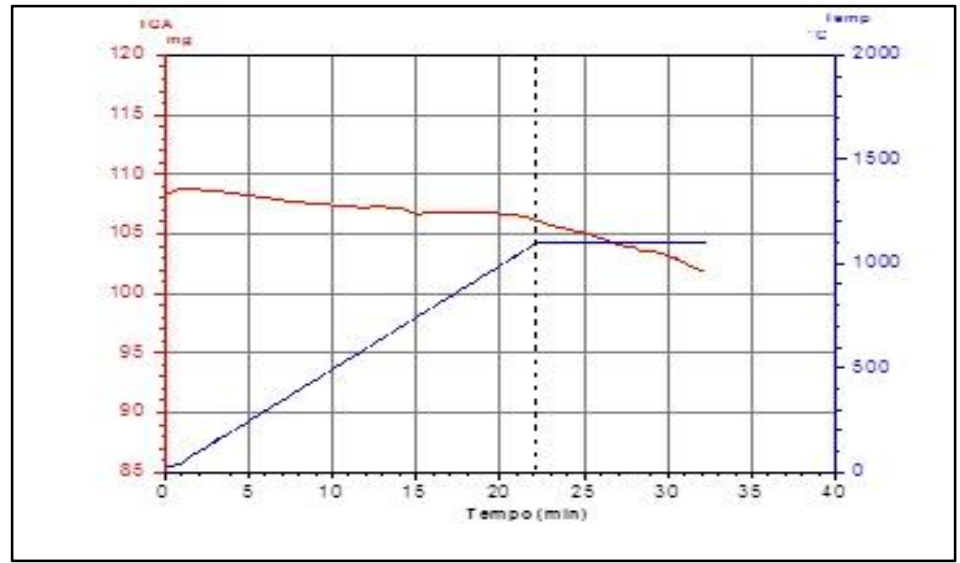

Figura 5 Termograma, $\mathrm{T}: 1100^{\circ} \mathrm{C}$, atm: $\mathrm{CO}_{2}$ e taxa de aquecimento $50^{\circ} \mathrm{C} / \mathrm{min}$.

\subsection{Resistência à compressão}

Os resultados de resistência à compressão mostrados na Figura 6, indicaram pequenas diferenças entre os briquetes autorredutores F-G, 50-50 e 70-30, além de exibirem valores crescentes a medida que o tempo de cura aumentou. Assim, aos 28 dias de cura a resistência máxima foi atingida, alcançando $207,9 \mathrm{~g}^{*} / \mathrm{cm}^{2}$. Tais valores, menores que dos briquetes sem o pó de coletor, se deveu ao fato deste material interferir na formação do gel gerado pelo cimento ARI que, precisando de água para endurecer, não conseguiu construir uma matriz mais resistente.

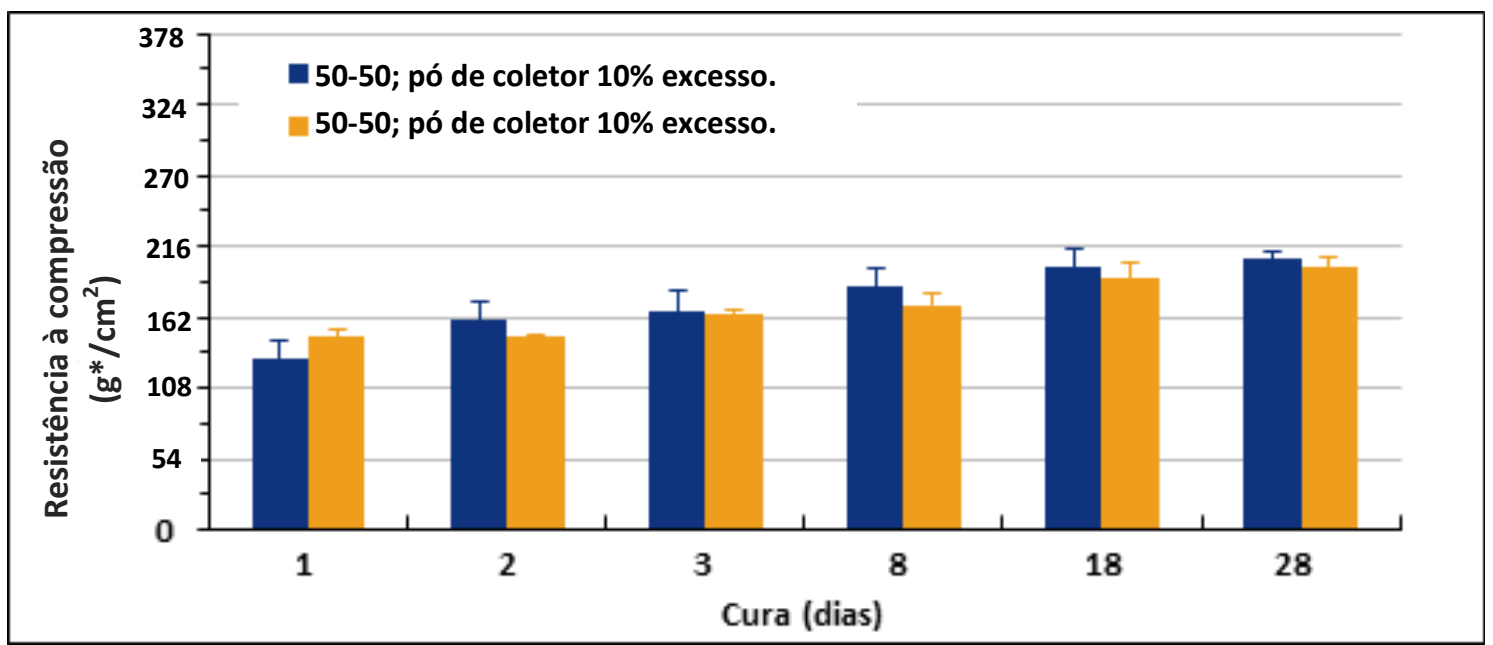

Figura 6 Resultados de resistência à compressão com o cimento ARI

\subsection{Metalização.}

As curvas de conversão metálica com o tempo e as de ajuste do modelo cinético de reação continua, são mostradas nas Figuras de 7 a 10, para os briquetes autorredutores F-G nas proporções $50-50$ e $70-30 \%$ e pó de coletor nas quantidades estequiométrica e com $10 \%$ de excesso.

Os resultados mostraram que as conversões metálicas aumentaram com a variação da temperatura e com a quantidade de pó do coletor. O briquete autorredutor F-G, na proporção $70-30$, foi o que alcançou a maior conversão metálica, $95 \%$, a $1150{ }^{\circ} \mathrm{C}$ com $10 \%$ de excesso de pó de coletor (Figura 10.a) 
O modelo cinético utilizado na análise dos dados dos ensaios de metalização, que resultou nas mais altas correlações (média de 0,995), foi o representado pela equação,

$$
-\ln (1-\chi)=\kappa t
$$

Onde $\chi$ é a conversão, $\kappa$ a constante cinética e t o tempo.

Os valores obtidos para $\kappa$, em $\mathrm{min}^{-1}$, para os experimentos estudados, estão indicados na tabela 5:

Tabela 5 Valores de $\kappa$, em $\min ^{-1}$

\begin{tabular}{|c|c|c|c|}
\hline \multicolumn{2}{|c|}{ Condições } & \multicolumn{2}{c|}{ Valores de $\kappa, \mathrm{min}^{-1}$} \\
\hline F-G, \% & ${\text { Temperatura, }{ }^{\circ} \mathrm{C}}^{2}$ & Briquet. esteq & Briquet. Esteq+10\% \\
\cline { 2 - 4 } \multirow{2}{*}{$50-50$} & 1100 & 0,0323 & 0,0445 \\
\hline \multirow{2}{*}{$70-30$} & 1150 & 0,0367 & 0,0554 \\
\cline { 2 - 4 } & 1100 & 0,0454 & 0,0613 \\
\hline
\end{tabular}
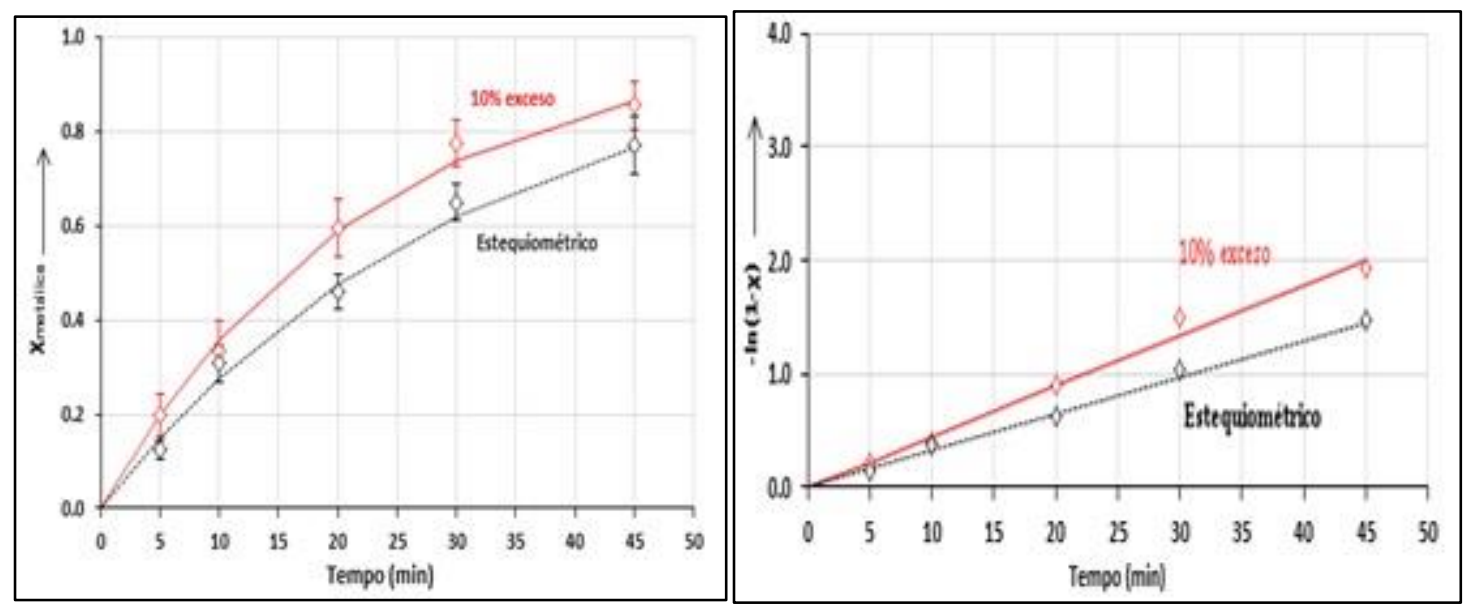

Figura 7: a) Conversão metálica b) modelo reação continua do briquete autorredutor, 50-50\% - pó de coletor (estequiométrico e $10 \%$ excesso), $\mathrm{T}: 1100^{\circ} \mathrm{C}$.
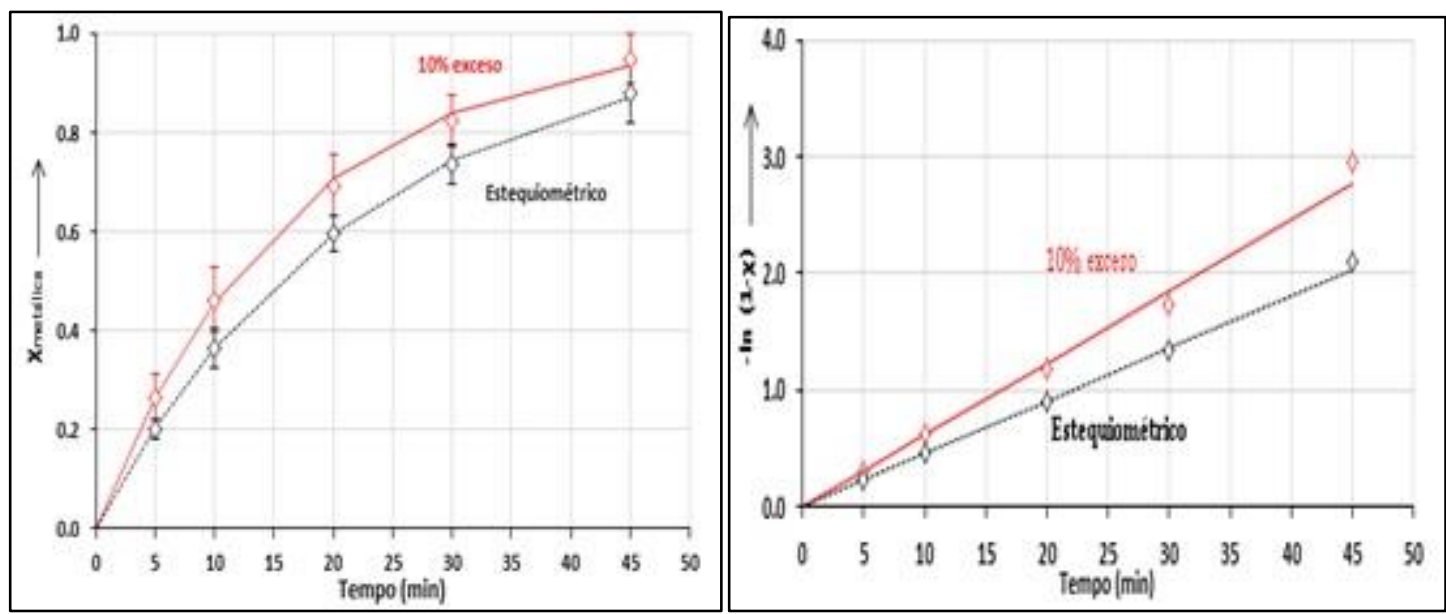

Figura 8: a) Conversão metálica b) modelo reação continua do briquete autorredutor, 70-30\% - pó de coletor (estequiométrico e $10 \%$ excesso), $\mathrm{T}: 1100^{\circ} \mathrm{C}$ 

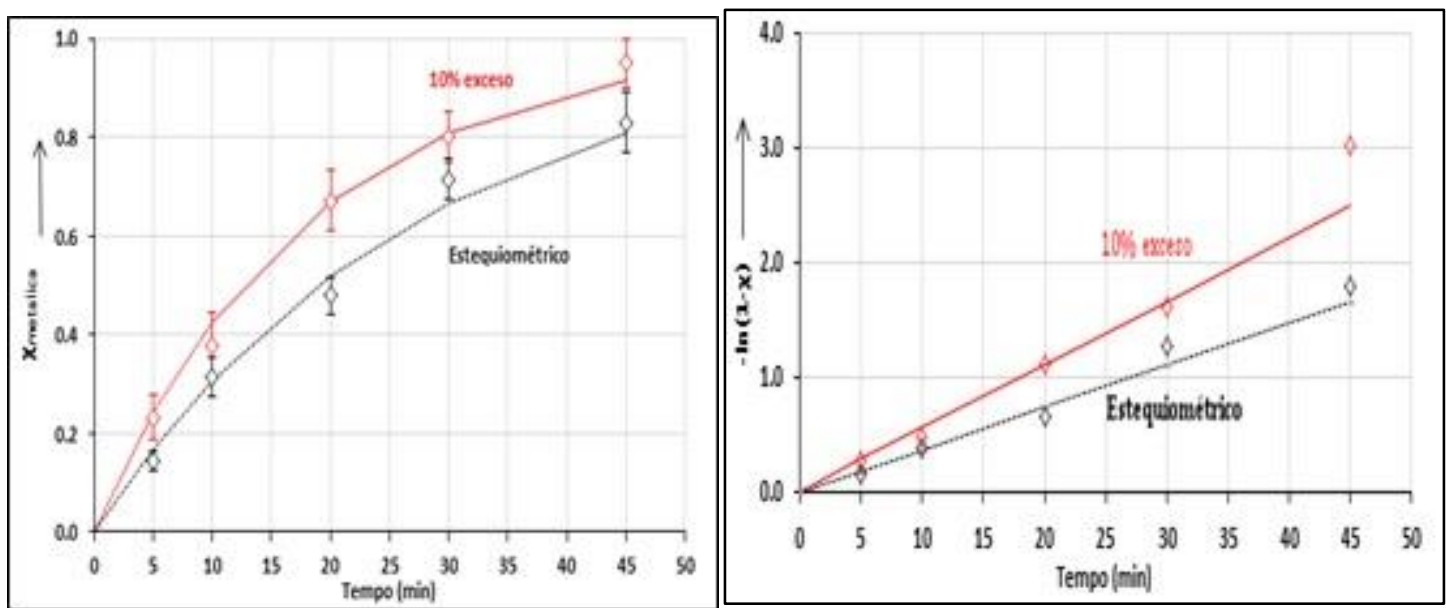

Figura 9: a) Conversão metálica b) modelo reação continua do briquete autorredutor, 50-50\% - pó de coletor (estequiométrico e $10 \%$ excesso), $\mathrm{T}: 1150^{\circ} \mathrm{C}$
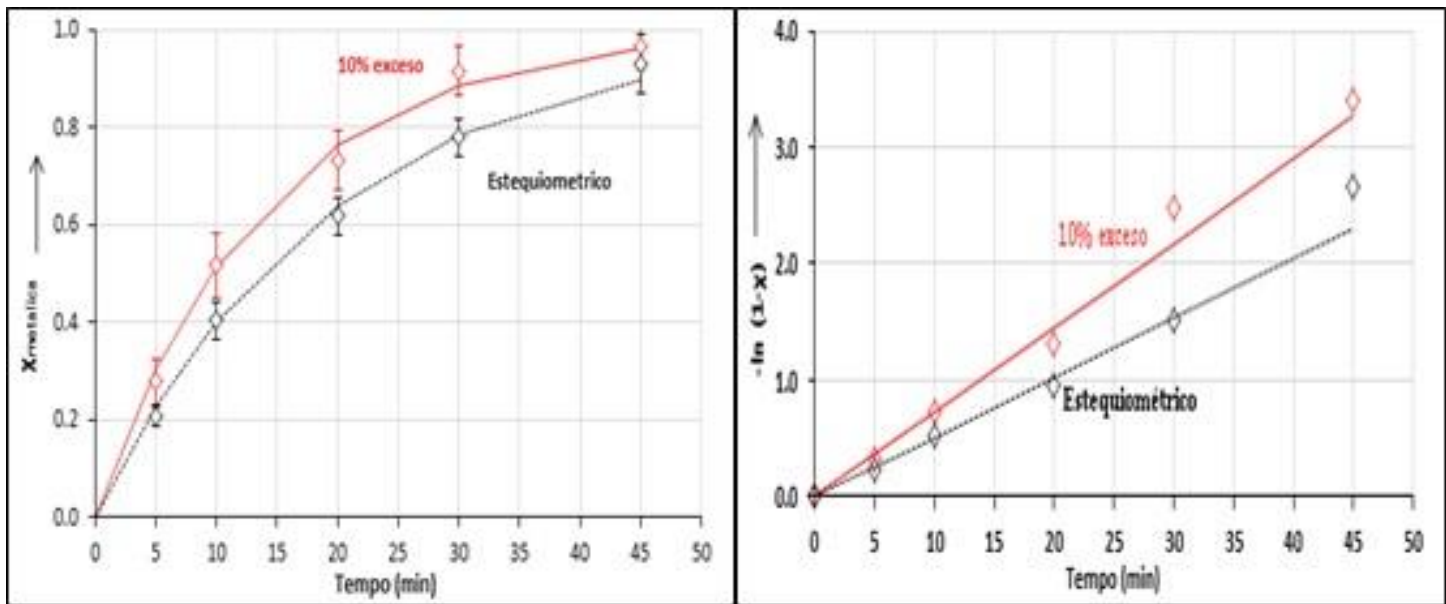

Figura 10: a) Conversão metálica b) modelo reação continua, do briquete autorredutor, $70-30 \%$ - pó de coletor (estequiométrico e $10 \%$ excesso), T: $1150^{\circ} \mathrm{C}$

\subsection{Poder refrigerante}

Adotando-se, em base termodinâmica, a sequência de reações mostrada na Tabela 6 e na Figura 11, para o sistema briquete autorredutor - aço liquido, quando estas fases entram em contato e considerando os potenciais de oxigênio da escoria calculado nas temperaturas de $1500^{\circ} \mathrm{C}$ e $1600^{\circ} \mathrm{C}$, iguais a $-321,2$ e $-308,0 \mathrm{~kJ} / \mathrm{mol}$, respectivamente, e o do banho metálico, nas mesmas temperaturas, iguais a $-349,4$ e $-353,8 \mathrm{~kJ} / \mathrm{mol}$, foi possível confirmar que nestes níveis de potenciais todas as reações de redução se viabilizaram. 
Tabela 6 Dados termodinâmicos e Sequência das reações : briquete autorredutor

\begin{tabular}{|c|c|c|c|}
\hline Sequência & Reações & $\Delta \mathrm{G}^{\circ}, \mathrm{kJ} / \mathrm{mol}$ & Temperatura, ${ }^{\circ} \mathrm{C}$ \\
\hline 1 & $2 / 3 \mathrm{Fe} 2 \mathrm{O} 3(\mathrm{~s})+2 / 3 \mathrm{C}(\mathrm{s})=4 / 3 \mathrm{FeO}(\mathrm{s})+2 / 3 \mathrm{CO}(\mathrm{g})$ & $114,8-0,138 \mathrm{~T}$ & $\theta<1371$ \\
\hline 2 & $2 \mathrm{FeO}(\mathrm{s})+2 \mathrm{C}(\mathrm{s})=2 \mathrm{Fe}(\mathrm{s})+2 \mathrm{CO}(\mathrm{g})$ & $298,6-0,300 T$ & $\theta<1371$ \\
\hline 3 & $2 \mathrm{FeO}(\mathrm{l})+2 \mathrm{C}(\mathrm{s})=2 \mathrm{Fe}(\mathrm{s})+2 \mathrm{CO}(\mathrm{g})$ & $194,6-0,269 T$ & $1371<\theta<1535$ \\
\hline \multirow[t]{2}{*}{3.1} & $2 \mathrm{FeO}(\mathrm{l})+2 \underline{\mathrm{C}}(\mathrm{s})=2 \mathrm{Fe}(\mathrm{s})+2 \mathrm{CO}(\mathrm{g})$ & $222,2-0.254 T$ & $\theta>1535$ \\
\hline & $2 \mathrm{Fe}_{(\mathrm{s})}=2 \mathrm{Fe}_{(l)}$ & $151,9-, 185 T$ & $1371<\theta<1535$ \\
\hline 4 & $2 \mathrm{FeO}_{(l)}+2 \mathrm{C}_{(\mathrm{s})}=2 \mathrm{Fe}_{(I)}+2 \mathrm{CO}$ & $13,8+0,007 T$ & $\theta=1535$ \\
\hline 4.1 & $2 \mathrm{FeO}_{(I)}+2[\underline{\mathrm{C}}]=2 \mathrm{Fe}_{(I)}+2 \mathrm{CO}$ & $179,5-0,170 \mathrm{~T}$ & $\theta>1535$ \\
\hline
\end{tabular}

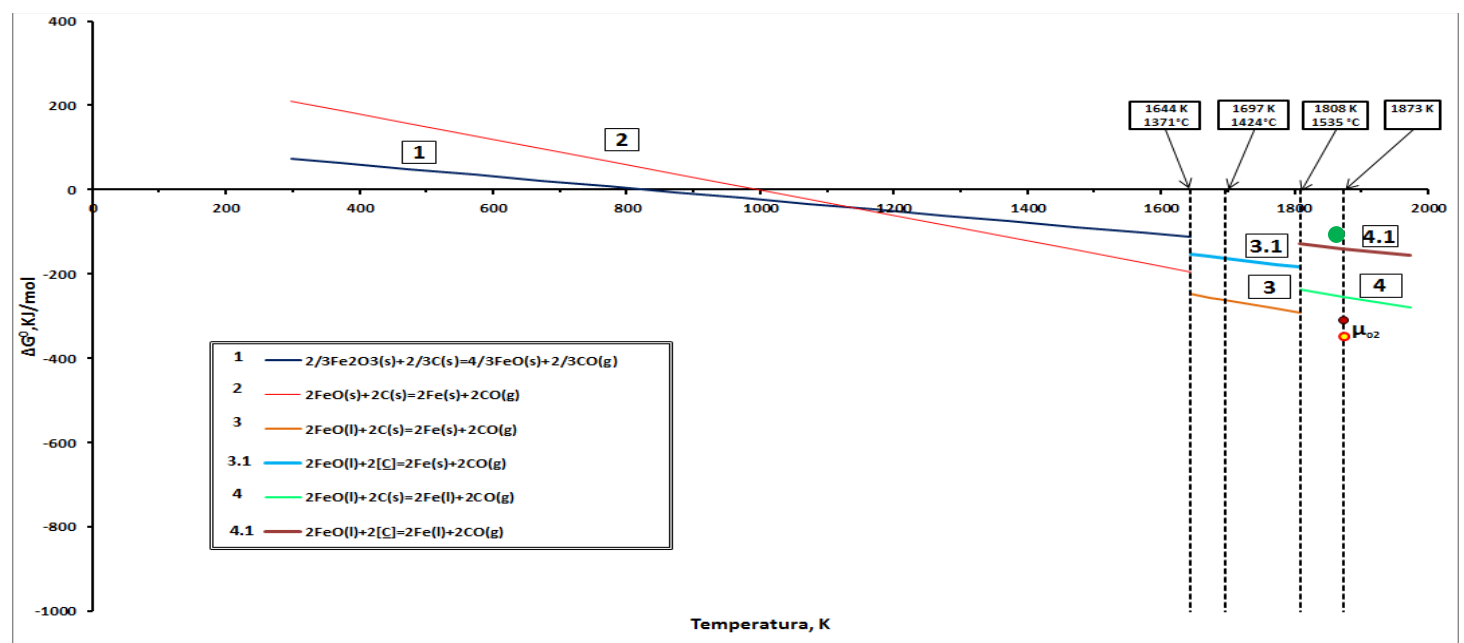

Figura 11 Sequencia das reações na redução dos óxidos: briquete autorredutor

\subsection{Balanço de energia}

Considerando como temperatura de referencia $1600^{\circ} \mathrm{C}$, as tabelas 7 e 8 apresentam os itens principais das entalpias envolvidas.

Tabela 7 Entalpias referentes ao briquete autorredutor $50-50 \%$ e $10 \%$ excesso de pó de coletor

\begin{tabular}{lccc}
\hline \multicolumn{1}{c}{ Briquete autorredutor } & $\%$ & Entalpias, kcal & $\mathbf{k c a l} / \mathbf{k g}$ \\
\hline $\mathrm{Fe}$ metálico & 11,8 & 322,56 & 38,06 \\
\hline $\mathrm{FeO}$ & 14,7 & 858.75 & 126,24 \\
\hline $\mathrm{Fe} \mathrm{O}_{3}$ & 34,4 & 145,30 & 49,98 \\
\hline $\mathrm{CaO}$ & 11,1 & 344,14 & 38,20 \\
\hline Ganga (Silicato Dicálcico) & 10,1 & 439,48 & 44,39 \\
\hline Carbono & 13,0 & 3842,06 & 499,47 \\
\hline LOI & 4,9 & & \\
\cline { 1 - 3 }
\end{tabular}


Tabela 8 Entalpias referentes ao briquete autorredutor F-G 70-30\% e 10\% excesso de pó de coletor

\begin{tabular}{|c|c|c|c|}
\hline Briquete autorredutor & $\%$ & Entalpias, kcal & kcal/kg \\
\hline Fe metálico & 16,3 & 322,56 & 52,58 \\
\hline $\mathrm{FeO}$ & 11,5 & 858,75 & 98,76 \\
\hline $\mathrm{Fe}_{2} \mathrm{O}_{3}$ & 31,4 & 145,30 & 45,62 \\
\hline $\mathrm{CaO}$ & 13,5 & 344,14 & 46,46 \\
\hline Ganga (Silicato Dicálcico) & 11,6 & 439,48 & 50,98 \\
\hline Carbono & 11,7 & 3842,06 & 404,28 \\
\hline \multirow[t]{2}{*}{ LOI } & 4,0 & & \\
\hline & & & 698,68 \\
\hline
\end{tabular}

Considerando como referencia o poder refrigerante da sucata metálica, de $340 \mathrm{kcal} / \mathrm{kg}$, e os valores calculados para os briquetes autorredutores, os seguintes índices foram estabelecidos:

- briquete autorredutor F-G, 50-50, com 10\% de excesso: 2,06 vezes a sucata.

- briquete autorredutor F-G, 70-30, com 10\% de excesso: 2,34 vezes a sucata.

De acordo com experimentos preliminares realizados pelo grupo de siderurgia em forno piloto instrumentado, foi possível confirmar a forte capacidade refrigerante do briquete autorredutor, conforme mostrado na Figura 12.

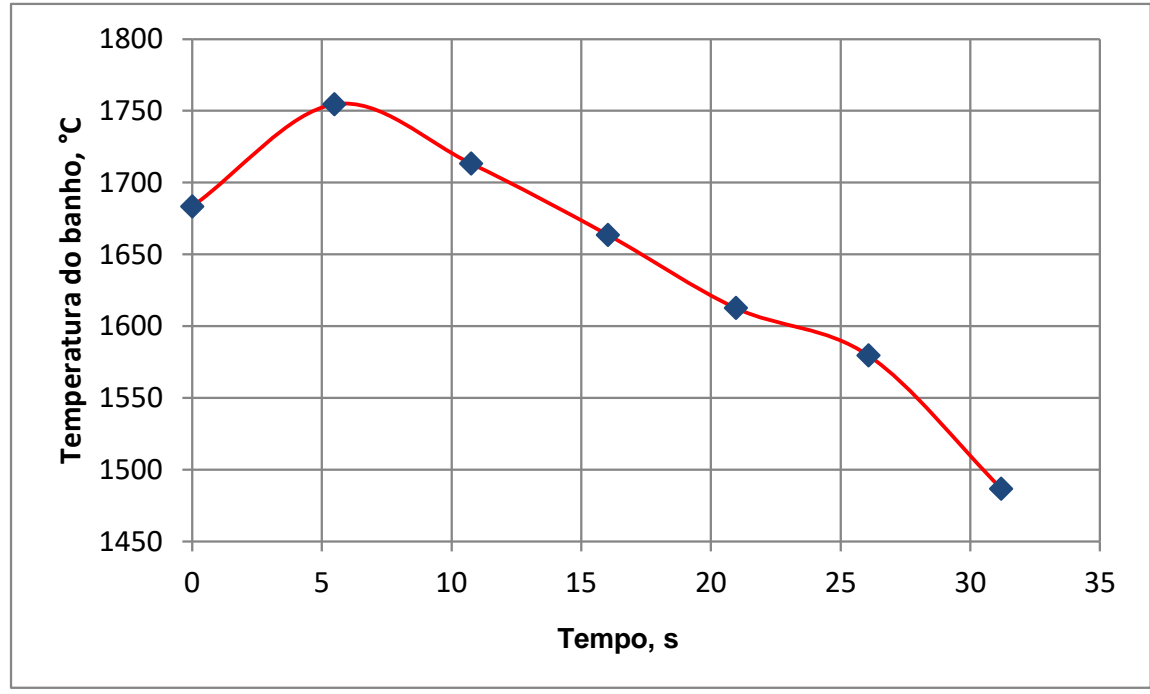

Figura 12 Efeito refrigerante de briquete autorredutor em aço liquido

\section{CONCLUSÃO}

- $\quad$ O tratamento prévio de estabilização volumétrica (passivação) dos pós-secos de aciaria foi imprescindível, no sentido de prevenir problemas de trincamento e fissura dos briquetes;

- Os briquetes autorredutores fabricados com cimento ARI atingiram resistências em 28 dias de cura praticamente independentes da proporção Fino - Grosso no briquete;

- Os ensaios TGA dos resíduos de aciaria passivados evidenciou perdas de massa compatíveis com o ganho alcançado durante o processo de passivação; 
- Os resultados obtidos nos ensaios ASTM (D-5341) e TGA, evidenciaram a presença de substancia carbonosa de alta reatividade, muito influenciada pela granulação fina;

- Nos testes de metalização em atmosfera de $\mathrm{N}_{2}$, a maior conversão, da ordem de $95 \%$, foi obtida com o briquete autorredutor F - G, $70-30$, a $1.150{ }^{\circ} \mathrm{C}$ e $10 \%$ de excesso de pó coletor;

- O mecanismo cinético de reação continua, $-\ln (1-x)=k t$, apresentou alta correlação, acima de 99\%, quando ajustado e calibrado com os dados experimentais;

- Com base no sequenciamento termodinâmico proposto para as reações de redução dos óxidos de ferro, foi possível confirmar, para os potenciais de oxigênio calculados com base na escoria e no banho, a viabilidade das reações de redução e a realização do balanço de energia.

Os índices estimados para o "poder refrigerante" dos briquetes autorredutores, foram:

i) Sucata:

ii) Briquete Autorredutor F - G (com $10 \%$ de excesso):

Proporção 50-50: $\quad 698,68 \mathrm{kcal} / \mathrm{kg}(2,05$ vezes à sucata)

Proporção 70-30: $\quad 798,33 \mathrm{kcal} / \mathrm{kg}$ (2,34vezes à sucata)

\section{Agradecimentos}

A CAPES pela bolsa de pós-graduação e ao grupo de siderurgia da PUC-Rio pelo apoio e realização dos experimentos.

\section{REFERÊNCIAS}

1 Union, European. JRC reference report. European Integrated Pollution Prevention and Control Bureau. Luxemburger: s.n., 2013. p. 627.

2 Contrucci, Noldin J. \& M. tecnored ironmaking process The Present and The Future. 2005. pp. 1-9.

3 Noldin, J. H., D’Abreu, J. C., \& Nogueira, P. F. Curso ABM de Novas Tecnologias de Ferro Primário. : ABM. São Paulo: s.n., 2009.

4 S. Kikuchl.ITmk3 Process. 2010, kobelco technology review, pp. 77-84.

5 Noldin J, Cotrucci M, Tecnored Process - High Potential in Using Different Kinds of Solid Fuels. .. Materials Research, pp. 447-451.

6 Martins K, D'Abreu J.C,. Morfologia do Ferro metálico em Briquetes autorredutores. Rio de Janeiro: Tese Mestrado, 2002.

7 D'Abreu, J. C.; Falero E.E.T,; Kohler, H.M; Bentes, M. A. G. ; Oliveira, L. F. R. . Briquetes Autorredutores de Residuos da Siderurgia Integrada: Reduções em Fase Sólida e Liquida. 45 Seminário de Redução de Minério de Ferro e Matérias Primas ABM-Week2015, 2015, Rio de Janeiro.

8 D'Abreu J.C \& R.N. Rodrigues Filho. Contribuição ao estudo da aglomeração de fino utilizando Cimento ARI, Cimento AL-61 e Cal hidratada. 5-9, s.l. Tecnologia em Metalurgia e Materiais, (2004).

9 M. Singh \& B. Bjorkman Use of cement-bonded agglomerates as burden material for blast furnaces. 2003, the Minerals, Metals \& Materials Society.

10 A. Babich, D. Senk and H. W. Gudenau Effect of coke reactivity and nut coke on blast furnace operation.,. 2009, Ironmaking and Steelmaking, Vol. 36, pp. 222-229.

11 D-5341, ASTM. Measuring coke reactivity index (CRI) and coke strength after reaction (CSR). 2004. 